\title{
Diagnostic performance of MRI and US in suspicion of penile fracture
}

\author{
Paul Spiesecke ${ }^{1}$, Josef Mang ${ }^{2}$, Thomas Fischer ${ }^{1}$, Bernd Hamm ${ }^{1}$, Markus H. Lerchbaumer ${ }^{\wedge} \wedge$ \\ ${ }^{1}$ Department of Radiology, Charité-Universitätsmedizin Berlin, corporate member of Freie Universität Berlin, Humboldt-Universität zu Berlin, \\ Berlin, Germany; ${ }^{2}$ Department of Urology, Charité-Universitätsmedizin Berlin, corporate member of Freie Universität Berlin, Humboldt- \\ Universität zu Berlin, Berlin, Germany \\ Contributions: (I) Conception and design: P Spiesecke, MH Lerchbaumer; (II) Administrative support: P Spiesecke, MH Lerchbaumer; (III) Provision \\ of study materials or patients: P Spiesecke, MH Lerchbaumer; (IV) Collection and assembly of data: P Spiesecke, MH Lerchbaumer; (V) Data \\ analysis and interpretation: P Spiesecke, MH Lerchbaumer; (VI) Manuscript writing: All authors; (VII) Final approval of manuscript: All authors. \\ Correspondence to: Markus H. Lerchbaumer, MD. Department of Radiology, Charité-Universitätsmedizin Berlin, Campus Charité Mitte, Charitéplatz \\ 1, 10117 Berlin, Germany. Email: markus.lerchbaumer@charite.de.
}

Background: Penile fracture (PF) is defined as rupture of the tunica albuginea of the corpora cavernosa. While most authors agree that rapid surgical therapy of this rare pathology leads to the best patient outcome, the role of imaging is highly controversial in the published literature. To obtain further evidence concerning the diagnostic accuracies of magnetic resonance imaging (MRI) and ultrasound (US) in the diagnostic assessment of patients with suspected PF.

Methods: We systematically reviewed MRI and US examinations performed in our institution between 2000 and 2021 and correlated imaging reports with either intraoperative finding or final clinical diagnosis. Inclusion criteria were: (I) patient age $\geq 18$ years, (II) examination between 2000 and 2021, (III) information available on patient's history and clinical presentation, and (IV) documented final diagnosis in discharge letter. Next to diagnostic accuracy, we describe typical imaging findings such as penile hematoma, tear of the tunica albuginea including location in terms of side and shaft segment affected, and involvement of corpus spongiosum.

Results: Overall, 46 of 88 included patients (54.5\%) had a confirmed diagnosis of PF. A total of 69 MRI and 31 US examinations were included. Sensitivity and specificity were 91.9\% (95\% CI: 78.7-97.2\%) and $90.6 \%$ (95\% CI: $75.8-96.8 \%$ ) for MRI and 71.4\% (95\% CI: 45.4-88.3\%) and 100.0\% (95\% CI: 81.6-100.0\%) for US, respectively.

Conclusions: The results of the present study suggest that MRI is more suitable to confirm PF and identify the site of the associated tunica albuginea tear while US is a good tool for ruling out PF.

Keywords: Diagnostic accuracy; imaging; magnetic resonance imaging (MRI); penile fracture (PF); ultrasound (US)

Submitted Oct 29, 2021. Accepted for publication Dec 22, 2021.

doi: 10.21037/tau-21-957

View this article at: https://dx.doi.org/10.21037/tau-21-957

\section{Introduction}

Penile fracture $(\mathrm{PF})$ is a rare urologic emergency that is characterized by a rupture of the tunica albuginea enclosing the corpora cavernosa due to blunt trauma $(1,2)$. Fracture is typically the result of an injury during intercourse or manipulation (3), causing, in most cases, a unilateral tear of the tunica albuginea $(1,4)$. Common clinical symptoms are hematoma, detumescence, fracture sound, and pain (4).

^ ORCID: 0000-0003-1151-9935. 
Onomatopoetically, the sound is described as cracking or snapping $(1,4)$.

Additionally, PF can be complicated by urethral laceration, noted clinically by urethral bleeding and urinary retention; however, absence of these symptoms does not rule out this important complication (1). Koifman et al. reported a mean intraoperative lesion size in the tunica albuginea of $1.5 \mathrm{~cm}$ and concomitant urethral trauma in $16 \%$ of cases (2).

The role of imaging in patients with suspected $\mathrm{PF}$ is controversial with some authors assuming that $\mathrm{PF}$ is mainly a clinical diagnosis and should be treated surgically without delay (2-4). Others report good diagnostic accuracies for magnetic resonance imaging (MRI) and ultrasound (US) $(5,6)$. Next to the detection of clinically false positive cases, presurgical imaging can reduce the surgical trauma: since most PFs are located in the proximal part of the penis, many cases are accessible via a minimal penoscrotal surgical approach, which is much less traumatic than complete degloving-if the lesion is diagnosed and characterized presurgically by imaging (7). Another important role of imaging in suspected $\mathrm{PF}$ is to rule out an injury of the dorsal vein, which can present with similar symptoms as $\mathrm{PF}$ but does not require emergency surgery (2).

In a systematic review, Falcone et al. conclude that US plays an important role in confirming the diagnosis and better defining the lesion site (1). While MRI showed higher accuracy than US, it was found to be hampered by low availability in emergency situations (1).

Therefore, the aim of this study is to directly compare the diagnostic accuracy of MRI and US when PF is suspected and to report typical imaging findings in a singlecenter setting.

We present the following article in accordance with the STARD reporting checklist (available at https://tau. amegroups.com/article/view/10.21037/tau-21-957/rc).

\section{Methods}

The study was conducted in accordance with the Declaration of Helsinki (as revised in 2013). The study was approved by institutional ethics committee of CharitéUniversitätsmedizin Berlin (No. EA2/307/20) and individual consent for this retrospective analysis was waived.

\section{Study population}

A systematic retrospective review of MRI and US examinations conducted for suspected PF was performed. Inclusion criteria were: (I) patient age $\geq 18$ years, (II) examination between 2000 and 2021, (III) information available on patient's history and clinical presentation, and (IV) confirmed final diagnosis retrieved from surgeon's report or discharge letter based on the International Classification of Diseases (ICD). The exclusion criterion was penile imaging requested for workup when another condition such as induratio penis plastica (IPP) was suspected.

Clinical records of the consecutive study patients were screened to extract information on the underlying mechanism of penile injury as well as symptoms and clinical findings including noticed sound, pain, hematoma, swelling, or penile deviation.

\section{Imaging analysis}

All patients underwent imaging in our hospital using standardized protocols. MRI protocols comprised T2weighted (w) standard 2D sequences and T1-w unenhanced $2 \mathrm{D}$ sequences in transverse and-based on positioning of the penis-sagittal or coronal plane. Contrast medium was administered as needed. In the patients assessed by US, a focused penile US with a high-frequency linear transducer and adequate presets available at the time of examination was performed.

All written diagnostic reports and stored images (if reports were inconclusive) were retrospectively analyzed by an experienced uroradiologist (MHL) blinded to the final diagnosis. For both MRI and US, the following findings were extracted from the detailed reports: penile hematoma, tear of the tunica albuginea enclosing the corpus cavernosum with localization (side and shaft segment), and involvement of the urethra or corpus spongiosum. A representative case of a PF depicted on MRI with visible hematoma on US (but no tunica tear) is shown in Figure 1. If no dedicated US examination was performed, urologic point-of-care US (POCUS) was used for comparative analysis of diagnostic accuracy.

The reported imaging-based diagnosis was recorded and compared with the intraoperative findings as gold standard 

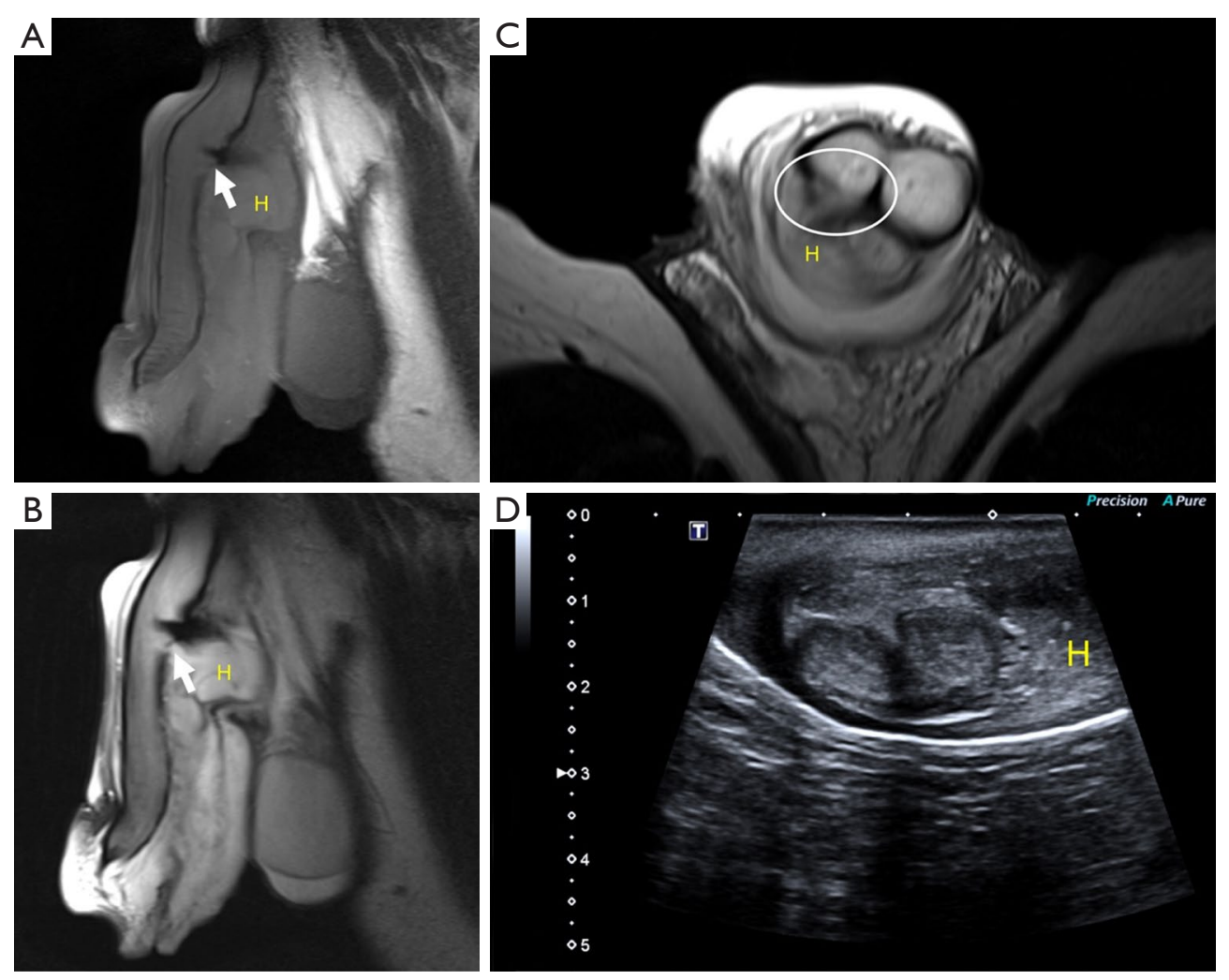

Figure 1 Confirmed diagnosis of penile fracture. (A,B) Nonenhanced MR T1w (A) and T2w (B) images in sagittal orientation reveal a focal tear (arrow) in the tunica albuginea of the right corpus cavernosum; (C) the tunica albuginea rupture is also visible in transverse orientation (white circle) in T2w image with surrounding local hematoma (H); (D) US demonstrates the local hematoma (H), while no clear tunica rupture was identified by the investigator. US, ultrasound.

for analysis of diagnostic accuracy. In patients not operated on, the final clinical diagnosis was used instead.

Additionally, a sub-analysis of the diagnostic accuracies including only the patients who received surgery was performed.

\section{Statistical analysis}

Continuous variables are reported as median and interquartile range (IQR). The reports of imaging findings and corresponding surgical and clinical reports of each patient were analyzed retrospectively for diagnostic accuracy by testing sensitivity, specificity, positive predictive value (PPV), and negative predictive value (NPV). The $\mathrm{chi}^{2}$ test was used to test cross tables of visible penile hematoma against final $\mathrm{PF}$ diagnosis.

All statistical analyses were performed using the SPSS software package (IBM Corp. Released 2019. IBM SPSS
Statistics for Windows, Version 26.0. Armonk, NY, USA: IBM Corp.).

\section{Results}

\section{Study population}

Overall, 88 patients were included in the study. Sixty-nine patients $(78.4 \%)$ were examined by MRI, and 25 patients (36.2\%) had a contrast-enhanced MRI examination. A dedicated penile US examination was performed in 31 (35.2\%) patients. Twelve study patients (13.6\%) underwent both MRI and US. In the subgroup without dedicated penile US, 17 patients underwent a urological POCUS examination, which was used for diagnostic accuracy analysis.

In 46 of the patients presenting with suspected PF $(52.3 \%)$, the diagnosis was confirmed as outlined above. 
Table 1 Baseline characteristics of the study population

\begin{tabular}{lc}
\hline Characteristics & $\mathrm{n} / \mathrm{N}(\%)$ \\
\hline Patient characteristics and trauma etiology & \\
Age (years) & 37 [28-45] \\
Etiology & \\
Sexual intercourse & $74 / 88(84.1 \%)^{\star}$ \\
Masturbation/manipulation & $8 / 88(9.1 \%)^{\star}$ \\
Other & $7 / 88(8.0 \%)$ \\
Documented details of history and clinical & \\
presentation & \\
Cracking sound & $32 / 88(36.4 \%)$ \\
Acute pain & $46 / 88(52.3 \%)$ \\
Penile hematoma & $65 / 88(73.9 \%)$ \\
Penile swelling & $53 / 88(60.2 \%)$ \\
Penile deviation & $13 / 88(14.8 \%)$ \\
Imaging examinations performed & \\
MRI & $32 / 88(36.4 \%)$ \\
US & $56 / 88(63.6 \%)$ \\
PocuS & $17 / 88(19.3 \%)$ \\
Surgery & \\
\hline Clinical-urological diagnosis & \\
\hline
\end{tabular}

Continuous variables are given as median [IQR], categorical variables as absolute/total numbers $(\mathrm{n} / \mathrm{N})$ with percentages in brackets. ${ }^{*}$, one patient reported simultaneous sexual intercourse and manipulation. MRI, magnetic resonance imaging; POCUS, point-of-care ultrasound; US, ultrasound.

Overall, 32 (36.4\%) patients were treated surgically. In two of them $(6.3 \%)$, no tear of the tunica albuginea of the corpora cavernosa was diagnosed intraoperatively. The most frequent clinical symptom was penile hematoma in 65 patients $(73.9 \%)$, followed by penile swelling (53 patients, $60.2 \%)$. The baseline characteristics of the study population are compiled in Table 1.

A total of 46 patients did not meet the inclusion criteria and were excluded-23 because clinical documentation was not available, 17 because they had other penile conditions, three because they were aged $<18$ years, two due to an inconclusive final clinical diagnosis, and one patient because he appeared to have recurrent PF and was already included with his first PF. The patient flow is illustrated in Figure 2.

\section{Imaging analysis}

Results for imaging findings are compiled in Table 2. As described in the methods section, we focused on penile hematoma, tear of the tunica albuginea of the corpora cavernosa and involvement of the corpus spongiosum. Overall, both modalities detected penile hematoma disproportionately often compared with the frequency of PFs.

Only one case of PF detected by MRI was bilateral. Right side unilateral PF was more common both in MRI and US, with $73.0 \%$ and $80.0 \%$ respectively. Urethral injury was detected by US in 3 cases $(9.7 \%$ ) and by MRI in 11 cases (15.9\%), respectively (Table 2).

No hematoma was found in $1 / 14(7.1 \%)$ finally positive cases (PF) in US and 2/37 (5.4\%) finally positive cases in MRI. Conversely, in the negative subgroup (no PF), penile hematoma was demonstrated in 12/17 (70.6\%) patients who underwent US and 13/32 (40.6\%) patients who underwent MRI. Therefore, $\mathrm{chi}^{2}$ tests of cross tables contrasting visibility of penile hematoma in imaging and final diagnosis revealed penile hematoma not to be a statistically significant indirect feature of $\mathrm{PF}$ in US $(\mathrm{P}=0.118)$ but in MRI $(\mathrm{P}<0.001)$.

\section{Diagnostic accuracy}

As described above, the present study defines PF as rupture of the tunica albuginea of the corpus cavernosum. Based on the imaging reports, $\mathrm{PF}$ was diagnosed in 37 patients by MRI and 10 patients by US. Matched with the diagnostic gold standard, MRI missed 3/37 (8.1\%) cases of confirmed PF and US missed 4/14 (28.6\%) cases, corresponding to an overall sensitivity of $91.9 \%$ (95\% CI: 78.7-97.2 \%) for MRI and $71.4 \%$ (95\% CI: $45.4-88.3 \%$ ) for US.

In the non-PF subgroup, MRI misclassified 3/32 (9.4\%) cases, whereas there was no false-positive case in the smaller US subgroup. The resulting specificities were $90.6 \%$ (95\% CI: $75.8-96.8 \%$ ) for MRI and $100.0 \%$ (95\% CI: 81.6-100.0\%) for US.

Moreover, urological POCUS, though performed in by far the smallest subgroup, also had high specificity of $100 \%$ (95\% CI: 67.6-100.0\%) but missed 7/9 PFs, resulting in a very low sensitivity of $22.2 \%$ (95\% CI: $6.3-54.7 \%$ ).

Diagnostic accuracies are compiled in Table 3. Subgroup analysis of the diagnostic performance of MRI with 44 cases $v s$. contrast-enhanced MRI (ceMRI) with 25 cases revealed no benefit of contrast medium administration. Sensitivities 


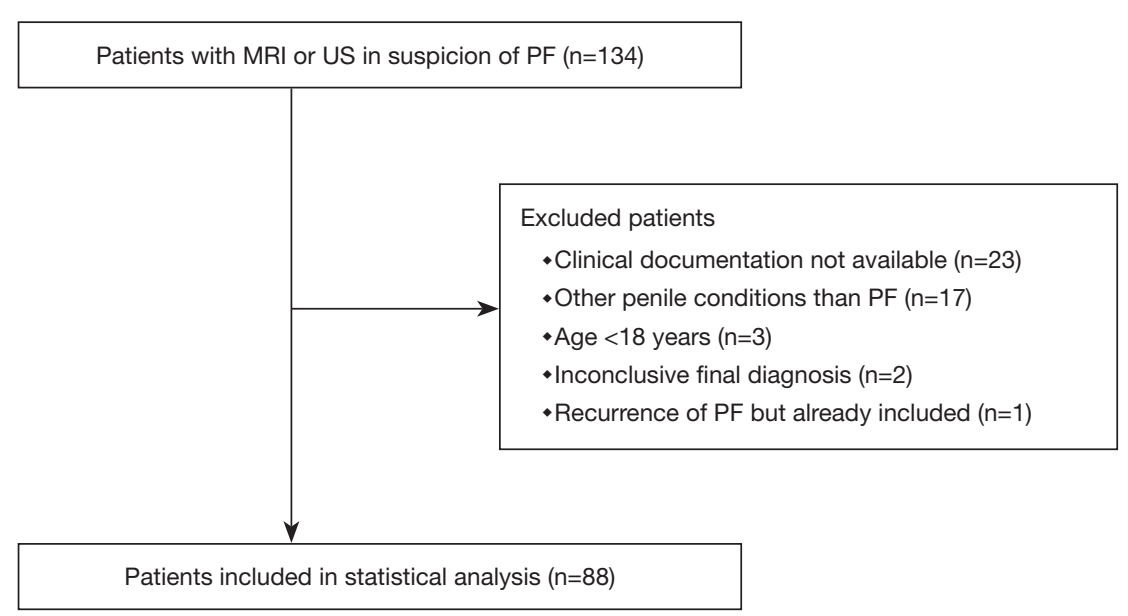

Figure 2 Flow of patients. The diagram illustrates the flow of patients who have been screened for study participation. MRI, magnetic resonance imaging; PF, penile fracture; US, ultrasound.

Table 2 Imaging findings in the study population

\begin{tabular}{lcc}
\hline Feature & US & MRI \\
\hline Penile hematoma & $25 / 31(80.6 \%)$ & $54 / 69(78.3 \%)$ \\
$\begin{array}{l}\text { Rupture of tunica albuginea } \\
\text { of corpora cavernosa }\end{array}$ & $10 / 31(32.2 \%)$ & $37 / 69(53.6 \%)$ \\
Side* & & \\
Left & $2 / 10(20.0 \%)$ & $9 / 37(24.3 \%)$ \\
Right & $8 / 10(80.0 \%)$ & $27 / 37(73.0 \%)$ \\
Bilateral & $0 / 10(0.0 \%)$ & $1 / 37(2.7 \%)$ \\
Shaft segment & & \\
Penile root & & $13 / 37(35.1 \%)$ \\
Mid-shaft & $7 / 10(70.0 \%)$ & $20 / 37(54.1 \%)$ \\
Apex & $3 / 10(30.0 \%)$ & $4 / 37(10.8 \%)$ \\
Involvement of corpus & $0 / 10(0.0 \%)$ & $11 / 69(15.9 \%)$ \\
spongiosum & $3 / 31(9.7 \%)$ & \\
Thereof with penile fracture & $0 / 3(0.0 \%)$ & $7 / 11(63.6 \%)$ \\
Thereof isolated & $3 / 3(100.0 \%)$ & $4 / 11(36.4 \%)$ \\
\hline Continuous varabs & &
\end{tabular}

Continuous variables are given as median (IQR), categorical variables as absolute/total numbers $(\mathrm{n} / \mathrm{N})$ with percentages in brackets. *, results for positive findings in imaging. MRI, magnetic resonance imaging; US, ultrasound.

and specificities were $92.0 \%$ vs. $91.7 \%$ and $94.7 \%$ vs. $84.6 \%$ for MRI vs. ceMRI, respectively.

Including operated patients $(\mathrm{n}=32)$ only, MRI $(\mathrm{n}=23)$ reached sensitivity of $95.5 \%$ (21 of 22 patients) and misdiagnosed one negative case. US $(n=11)$ reached sensitivity of $70.0 \%$ (7 of 10 patients) and correctly diagnosed one negative case.

Comparing the final diagnosis of US and MRI in patients $(\mathrm{n}=12)$ who underwent both imaging modalities, MRI and US showed congruence in 7 of 7 cases which were negatively reported in MRI, whereas in 2 of 5 patients who were reported to have PF in MRI, US described no present $\mathrm{PF}$ (accordance of $60.0 \%$ ).

An example of a proven $\mathrm{PF}$ with accurate imaging-based localization of the tear is presented in Figure 3.

\section{Discussion}

The major results of the present study can be summarized as follows: MRI has the highest sensitivity for diagnosing a $\mathrm{PF}$ and detecting the focal tear without much improvement in diagnostic accuracy after contrast agent administration. US has high specificity for ruling out a PF.

In an MRI study of 38 patients published in 2017, Saglam et al. found $100 \%$ sensitivity and $87.5 \%$ specificitytherefore showing a higher sensitivity than in our patients (6). The prospective study of Zare Mehrjardi et al. reported a detection rate of $100 \%$ of the surgically proven tears of the tunica albugina in 25 patients and thus confirmed the high diagnostic accuracy published by Saglam et al. $(5,6)$. Another investigation evaluating MRI of 28 patients with presumed $\mathrm{PF}$ also showed a high sensitivity of $100 \%$ and a moderate specificity of $77.8 \%$ (8). Therefore, external validity can be as assumed to be sufficient, although 
Table 3 Diagnostic performance of the imaging modalities analyzed in our study

\begin{tabular}{lcc}
\hline Imaging modality & Sensitivity & Specificity \\
\hline MRI & $34 / 37(91.9 \%, 95 \% \mathrm{Cl}: 78.7-97.2 \%)$ & $29 / 32(90.6 \%, 95 \% \mathrm{Cl}: 75.8-96.8 \%)$ \\
US & $10 / 14(71.4 \%, 95 \% \mathrm{Cl}: 45.4-88.3 \%)$ & $17 / 17(100.0 \%, 95 \% \mathrm{Cl}: 81.6-100.0 \%)$ \\
POCUS & $2 / 9(22.2 \%, 95 \% \mathrm{Cl}: 6.3-54.7 \%)$ & $8 / 8(100 \%, 95 \% \mathrm{Cl}: 67.6-100.0 \%)$ \\
\hline
\end{tabular}

Categorial variables as absolute/total numbers $(\mathrm{n} / \mathrm{N})$ with percentages in brackets. $\mathrm{Cl}$, confidence interval; MRI, magnetic resonance imaging; POCUS, point-of-care ultrasound; US, ultrasound.
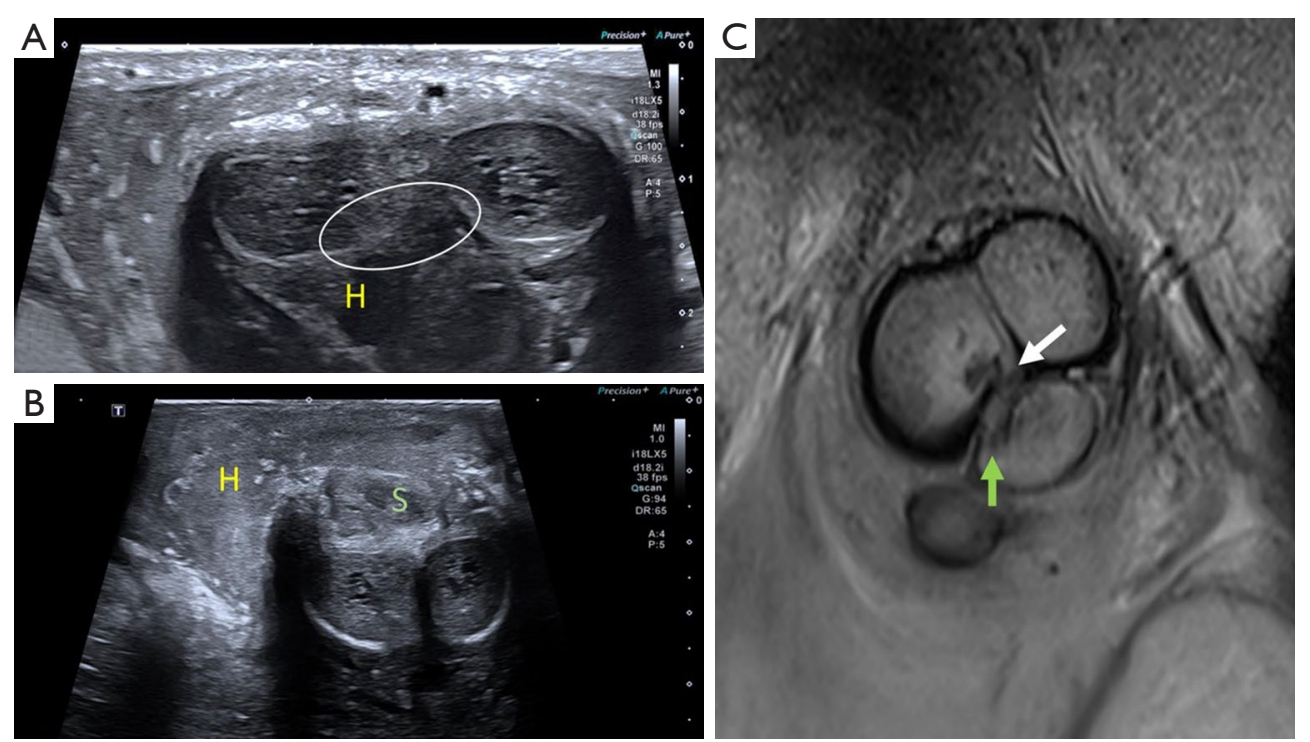

Figure 3 Confirmed penile fracture correctly identified by US and nonenhanced MRI. (A) High-resolution US with an 18-MHz linear transducer shows discontinuity of the hyperechogenic tunica albuginea of the right corpus cavernosum (white circle) with local hematoma $(\mathrm{H})$; (B) in different scan plane (transverse view), US reveals large surrounding hematoma (H) and blurred corpus spongiosum (S); (C) nonenhanced MRI (high-resolution T2w image in coronal orientation) reveals clear tunica rupture (white arrow) and hypointense spot in the right corpus cavernosum and involvement of the corpus spongiosum (green arrow), which was confirmed during surgical repair. US, ultrasound; MRI, magnetic resonance imaging.

we found lower sensitivity than in previous studies.

Fewer data are available on the diagnostic accuracy of US. Zare Mehrjardi et al. reported a detection rate of $88 \%$ for US, which was lower than that of MRI (5). At the same time, the authors reported a lower confidence of preoperative lesion mapping (5). In a small group of 12 patients, Martí de Gracia et al. found $83 \%$ sensitivity and $100 \%$ specificity for US using intraoperative findings as the gold standard (9). Both studies show basically the same magnitude of diagnostic accuracy as in our study.

Generally, many authors emphasize the importance of prompt surgery for PF $(2-4,10)$. In a meta-analysis, Amer et al. found that surgery significantly improved outcome compared with conservative therapy (10). Furthermore, noted that the majority of authors of the included primary studies did not use any imaging at all, underlining the importance of the clinical examination (10).

If clinicians decide that imaging is needed before treatment, US should be preferred to MRI because it shortens the delay to subsequent surgery. With its high specificity, US is a reasonable option in patients with a low likelihood of PF. Also, US can be performed at the bedside and in emergency units, making it suitable for less mobile patients. Finally, US is much more widely available 
than MRI, especially in smaller hospitals or when patients present at night.

Imaging is helpful if the diagnosis is unclear, as also discussed by Phillips et al. (11). Another benefit of imaging is that it may reduce the surgical trauma by exactly locating the lesion and thus allowing less extensive surgical exploration and additionally, degloving regularly includes circumcision (7). This approach was for instance discussed in a case report of Rosi et al. (12). However, penile degloving remains the standard surgical procedure-as supported by evidence from a study of eight patients with PFs reported by Kamdar et al. (13). The authors emphasize the superior intraoperative overview of all spongy bodies with the option of intraoperative injection of coloring agent for the detection of initially overlooked lesions (13).

If imaging is performed, contrast medium should be used cautiously. Our findings suggest that there is no benefit of a contrast-enhanced series compared with nonenhanced imaging. Moreover, Zare Mehrjardi et al. accomplished precise mapping of the tear using standardized nonenhanced MRI (5). Furthermore, additional contrast-enhanced series account for a large proportion of the total costs of such an MRI examination, as for example reported in a recently published study investigating the imaging of renal cysts (14).

Contrast-enhanced US (CEUS) is mainly used in abdominal imaging-and only one case report of CEUS in PF exists so far (15). The authors emphasize advantages like more precise surgical planning and less extensive incision (15). Another case report describes a "microbubbleenhanced retrograde ultrasound urethrogram" that confirmed injury of the urethra avoiding radiation exposure of retrograde urethrography which is a standard procedure in inconclusive cases (16). Finally, both the intravenous or urethral application of ultrasound contrast agent (UCA) has not got achieved evidence so far.

The patients in our study were examined and reported by board-certified radiologists without subspecialization in urogenital radiology, which might have contributed to a lower diagnostic accuracy (especially on US), but, on the other hand, reflects the real-life situation when a patient with suspected PF seeks medical attention. Additionally, it must be taken into account that US technology has made dramatic advances, especially in terms of spatial resolution, since the first patients included in our retrospective analysis were examined. Therefore, state-of-the-art high-end US systems may achieve higher diagnostic accuracy than found in our study. Further improvement might be achieved by using multiparametric US (17). Possible benefits of techniques like US elastography in patients with suspected $\mathrm{PF}$ remain to be investigated.

Overall, US technologies used in clinical routine during the period under investigation here already turned out to be highly specific (Table 3) and are therefore suitable for ruling out PF. Of note, demonstration of hematoma by US did not turn out to be a statistically significant feature of PF. Therefore, a feature that could be helpful in selecting patients who should have an MRI for confirmation and surgery planning remains to be identified. Alternatively, future research might show that, with the image quality achieved using high-end US equipment, indirect imaging signs of $\mathrm{PF}$ are redundant.

A drawback of US is that a massive hematoma can mask the tunica albuginea tear, which may thus be overlooked (11). In such cases, a larger penetration depth must be chosen-vitiating the highest possible spatial resolution. This might alter the appearance of the tunica albuginea border as illustrated in Figure 1. Such appearances must be differentiated from scrotal hematoma, which can be massive, as illustrated in Figure 4. If there is good visualization of critical structures by US, many decisive diagnoses of course can be made. For instance, intracavernous hematoma has a typical US appearance, as outlined by Cozzi et al. Initially, these hematomas are hyperechoic and reshape as cystic formations over time (18). Hematoma can be treated conservatively as long as no rupture of the tunica albuginea is present (18).

\section{Limitations}

A major limitation of our study is its retrospective design, although this is a rational approach when investigating a rare pathology such as PF. Explained by retrospective character of the investigation, missing clinical data caused more patient exclusions-nevertheless, we prefer more restrictive inclusion criteria to produce more valuable data.

\section{Conclusions}

Our findings show MRI to be clearly more sensitive than US in diagnosing PF and detecting the focal tear. Nevertheless, US rules out PF with high specificity. 

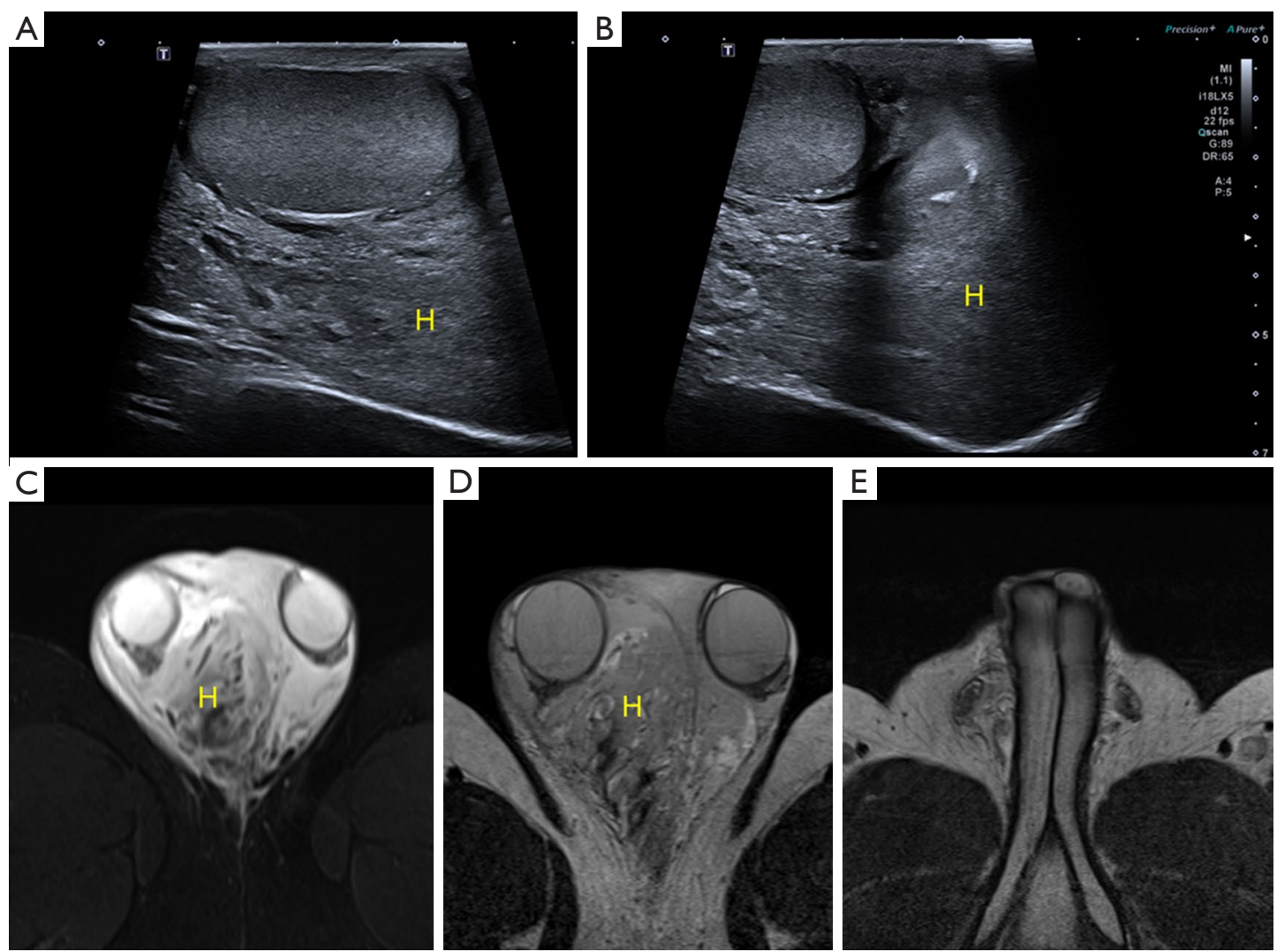

Figure 4 Patient with suspected penile fracture and large scrotal hematoma. (A,B) US reveals no tunica albuginea rupture (not shown) but large scrotal hematoma $(\mathrm{H})$; (C-E) clear identification of an acute scrotal hematoma $(\mathrm{H})$ on T1w $(\mathrm{C})$ and T2w images (D) without a rupture of the tunica albuginea (E, representative image). US, ultrasound.

\section{Acknowledgments}

The authors thank Ms. Bettina Herwig for language editing of the manuscript.

Funding: None.

\section{Footnote}

Reporting Checklist: The authors have completed the STARD reporting checklist. Available at https://tau.amegroups.com/ article/view/10.21037/tau-21-957/rc

Data Sharing Statement: Available at https://tau.amegroups. com/article/view/10.21037/tau-21-957/dss

Conflicts of Interest: All authors have completed the ICMJE uniform disclosure form (available at https://tau.amegroups. com/article/view/10.21037/tau-21-957/coif). The authors have no conflicts of interest to declare.
Ethical Statement: The authors are accountable for all aspects of the work in ensuring that questions related to the accuracy or integrity of any part of the work are appropriately investigated and resolved. The study was conducted in accordance with the Declaration of Helsinki (as revised in 2013). The study was approved by the institutional ethics committee of Charité-Universitätsmedizin Berlin (No. EA2/307/20) and individual consent for this retrospective analysis was waived.

Open Access Statement: This is an Open Access article distributed in accordance with the Creative Commons Attribution-NonCommercial-NoDerivs 4.0 International License (CC BY-NC-ND 4.0), which permits the noncommercial replication and distribution of the article with the strict proviso that no changes or edits are made and the original work is properly cited (including links to both the formal publication through the relevant DOI and the license). See: https://creativecommons.org/licenses/by-nc-nd/4.0/. 


\section{References}

1. Falcone M, Garaffa G, Castiglione F, et al. Current Management of Penile Fracture: An Up-to-Date Systematic Review. Sex Med Rev 2018;6:253-60.

2. Koifman L, Barros R, Júnior RA, et al. Penile fracture: diagnosis, treatment and outcomes of 150 patients. Urology 2010;76:1488-92.

3. Eke N. Fracture of the penis. Br J Surg 2002;89:555-65.

4. Barros R, Hampl D, Cavalcanti AG, et al. Lessons learned after 20 years' experience with penile fracture. Int Braz J Urol 2020;46:409-16.

5. Zare Mehrjardi M, Darabi M, Bagheri SM, et al. The role of ultrasound (US) and magnetic resonance imaging (MRI) in penile fracture mapping for modified surgical repair. Int Urol Nephrol 2017;49:937-45.

6. Saglam E, Tarhan F, Hamarat MB, et al. Efficacy of magnetic resonance imaging for diagnosis of penile fracture: A controlled study. Investig Clin Urol 2017;58:255-60.

7. De Luca F, Garaffa G, Falcone M, et al. Functional outcomes following immediate repair of penile fracture: a tertiary referral centre experience with 76 consecutive patients. Scand J Urol 2017;51:170-5.

8. Sokolakis I, Schubert T, Oelschlaeger M, et al. The Role of Magnetic Resonance Imaging in the Diagnosis of Penile Fracture in Real-Life Emergency Settings: Comparative Analysis with Intraoperative Findings. J Urol 2019;202:552-7.

9. Martí de Gracia M, Muñiz Iriondo I, García Fresnadillo JP, et al. Corpus cavernosum fracture: the ultrasound in

Cite this article as: Spiesecke P, Mang J, Fischer T, Hamm B, Lerchbaumer MH. Diagnostic performance of MRI and US in suspicion of penile fracture. Transl Androl Urol 2022;11(3): 377-385. doi: 10.21037/tau-21-957 the emergency diagnosis. Radiologia 2013;55:154-9.

10. Amer T, Wilson R, Chlosta P, et al. Penile Fracture: A Meta-Analysis. Urol Int 2016;96:315-29.

11. Phillips EA, Esposito AJ, Munarriz R. Acute penile trauma and associated morbidity: 9-year experience at a tertiary care center. Andrology 2015;3:632-6.

12. Rosi G, Fontanella P, Venzi G, et al. 3T MR-guided minimally-invasive penile fracture repair. Arch Ital di Urol Androl organo Uff [di] Soc Ital di Ecogr Urol e Nefrol 2016;88:68-9.

13. Kamdar C, Mooppan UMM, Kim H, et al. Penile fracture: preoperative evaluation and surgical technique for optimal patient outcome. BJU Int 2008;102:1640-4.

14. Spiesecke P, Reinhold T, Wehrenberg Y, et al. Costeffectiveness analysis of multiple imaging modalities in diagnosis and follow-up of intermediate complex cystic renal lesions. BJU Int 2021;128:575-85.

15. Panayiotou A, Huang DY, Makanjuola JK, et al. Penile fracture: added value of novel assessment with contrastenhanced ultrasound. BMJ Case Rep 2019;12:230864.

16. Czarnecki O, von Stempel CB, Sangster P, et al. Microbubble-enhanced ultrasound to demonstrate urethral transection in a case of penile fracture. BMJ Case Rep 2017;2017:bcr-2017-220073.

17. Sidhu PS. Multiparametric Ultrasound (MPUS) Imaging: Terminology Describing the Many Aspects of Ultrasonography. Ultraschall Med 2015;36:315-7.

18. Cozzi D, Verrone GB, Agostini S, et al. Acute penile trauma: imaging features in the emergency setting. Radiol Med 2019;124:1270-80. 